\title{
Systematic error cancellation for the PIXIE four-port interferometric polarimeter
}

\author{
Alan Kogut, Dale J. Fixsen
}

Alan Kogut, Dale J. Fixsen, "Systematic error cancellation for the PIXIE fourport interferometric polarimeter," Proc. SPIE 10708, Millimeter, Submillimeter, and Far-Infrared Detectors and Instrumentation for Astronomy IX, $107083 \mathrm{U}$ (9 July 2018); doi: 10.1117/12.2311503

Event: SPIE Astronomical Telescopes + Instrumentation, 2018, Austin, Texas, United States 


\title{
Systematic error cancellation for the PIXIE four-port interferometric polarimeter
}

\author{
Alan Kogut ${ }^{\mathrm{a}}$ and Dale J. Fixsen ${ }^{\mathrm{a}, \mathrm{b}}$

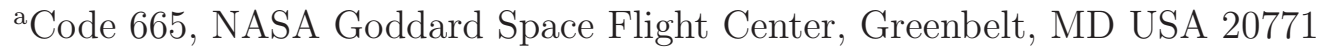 \\ ${ }^{\mathrm{b}}$ University of Maryland, College Park MD USA
}

\begin{abstract}
The Primordial Inflation Explorer (PIXIE) is an Explorer-class mission concept to measure the gravitational-wave signature of primordial inflation through its distinctive imprint on the linear polarization of the cosmic microwave background. Its optical system couples a polarizing Fourier transform spectrometer to the sky to measure the differential signal between orthogonal linear polarization states from two co-pointed beams on the sky. The double differential nature of the four-port measurement mitigates beam-related systematic errors common to the two-port systems used in most CMB measurements. We describe the polarized beam patterns for PIXIE and assess the systematic error for measurements of CMB polarization.
\end{abstract}

Keywords: cosmic microwave background, systematic error, beam patterns, polarimeter, Fourier transform spectrometer

\section{INTRODUCTION}

Polarization of the cosmic microwave background (CMB) provides a powerful test of the physics of the early universe. An arbitrary pattern of linear polarization may be decomposed into a symmetric component (even parity E-modes) and an anti-symmetric component (odd parity B-modes). Scalar sources such as temperature or density perturbations can only source even-parity E-modes, while gravitational waves created during an inflationary epoch in the early universe can source either parity. Detection of the B-mode signal in the CMB polarization field is thus recognized as a "smoking gun" signature of inflation, testing physics at energies inaccessible through any other means. ${ }^{1-8}$

The amplitude of the gravitational wave signal depends on the energy scale of inflation as

$$
E=1.06 \times 10^{16}\left(\frac{r}{0.01}\right)^{1 / 4} \mathrm{GeV}
$$

where $r$ is the power ratio of gravitational waves to density fluctuations. ${ }^{9}$ In most large-field models, $r$ is predicted to be of order 0.01, corresponding to polarized amplitude $30 \mathrm{nK}$ or energy near the Grand Unified Theory scale, $10^{16} \mathrm{GeV}$. Signals at this amplitude could be detected by a dedicated polarimeter, providing a critical test of a central component of modern cosmology. Detection of a gravitational-wave component in the CMB polarization would have profound implications for both cosmology and high-energy physics. It would establish that inflation actually occurred, provide a direct, model-independent determination of the relevant energy scale, and test physics at energies a trillion times beyond those accessible to particle accelerators. Generation of gravitational waves during inflation is purely a quantum-mechanical process: a detection of the B-mode signal provides direct observational evidence that gravity obeys quantum mechanics.

Characterizing CMB polarization at parts-per-billion accuracy requires careful control of systematic errors. A particular concern are systematic errors related to the instrument optics, which can couple the much brighter unpolarized temperature fluctuations into a false polarization signal. All CMB instruments must couple the detectors to the sky, and must therefore account for potential beam-related systematic errors. An extensive literature discusses common effects and mitigation strategies. ${ }^{10-13}$

The Primordial Inflation Explorer (PIXIE) is an Explorer-class mission designed to measure the inflationary signature in polarization as well as blackbody spectral distortions from more recent cosmological epochs. ${ }^{14}$ PIXIE

Send correspondence to: Alan.J.Kogut@nasa.gov; telephone 0013012860853

Millimeter, Submillimeter, and Far-Infrared Detectors and Instrumentation for Astronomy IX, edited by Jonas Zmuidzinas, Jian-Rong Gao, Proc. of SPIE Vol. 10708, 107083U

(c) 2018 SPIE · CCC code: 0277-786X/18/\$18 - doi: 10.1117/12.2311503

Proc. of SPIE Vol. $10708107083 \mathrm{U}-1$ 
differs from most CMB polarimeters in its use of a polarizing Fourier transform spectrometer coupled to the sky through a multi-moded optical system. The double differential nature of the resulting four-port measurement minimizes beam-related systematic errors common to the two-port systems used in most CMB measurements. We describe the polarized beam patterns for PIXIE and assess the systematic error for measurements of CMB polarization.

\section{PIXIE OPTICAL SYSTEM}

The PIXIE optical system may be described as a four-port device (Fig 1). Reflective foreoptics couple a polarizing Fourier Transform Spectrometer to the sky. The FTS introduces an optical phase delay between the two input beams, and routes recombined beams to non-imaging concentrators at each of two output ports. Within each concentrator, a pair of polarization-sensitive detectors measure the power as a function of optical phase delay. Let $\vec{E}=E_{x} \hat{x}+E_{y} \hat{y}$ represent the electric field incident from the sky. The power $P$ at the detectors as a function of the mirror position $z$ may be written

$$
\begin{aligned}
& P_{L x}=1 / 2 \int\left\{\left(E_{A x}^{2}+E_{B y}^{2}\right)+\left(E_{A x}^{2}-E_{B y}^{2}\right) \cos (4 z \omega / c)\right\} d \omega \\
& P_{L y}=1 / 2 \int\left\{\left(E_{A y}^{2}+E_{B x}^{2}\right)+\left(E_{A y}^{2}-E_{B x}^{2}\right) \cos (4 z \omega / c)\right\} d \omega \\
& P_{R x}=1 / 2 \int\left\{\left(E_{A y}^{2}+E_{B x}^{2}\right)+\left(E_{B x}^{2}-E_{A y}^{2}\right) \cos (4 z \omega / c)\right\} d \omega \\
& P_{R y}=1 / 2 \int\left\{\left(E_{A x}^{2}+E_{B y}^{2}\right)+\left(E_{B y}^{2}-E_{A x}^{2}\right) \cos (4 z \omega / c)\right\} d \omega,
\end{aligned}
$$

where $\mathrm{L}$ and $\mathrm{R}$ refer to the detectors in the left and right concentrators, A and B refer to the two input beams, $\omega$ is the angular frequency of incident radiation, $z$ is the mirror position, and the factor of 4 reflects the symmetric folding of the optical path. When both input ports are open to the sky, the power at each detector consists of a dc term proportional to the intensity (Stoke I) plus a term modulated by the mirror position $z$, proportional to the linear polarization (Stokes Q) in instrument-fixed coordinates. Rotation of the instrument about the beam axis rotates the instrument coordinate system relative to the sky to allow separation of Stokes $Q$ and $U$ parameters on the sky.

Rotation of the instrument relative to the sky can produce systematic errors in the recovered polarization if the instrument beams are not azimuthally symmetric. This effect has been well studied for two-port devices which couple the sky directly to a single polarization-sensitive detector. The dominant systematic error for such a device is temperature-polarization coupling as the beam ellipticity interacts with local gradients in the unpolarized sky intensity, producing a spin-dependent signal degenerate with true polarization.

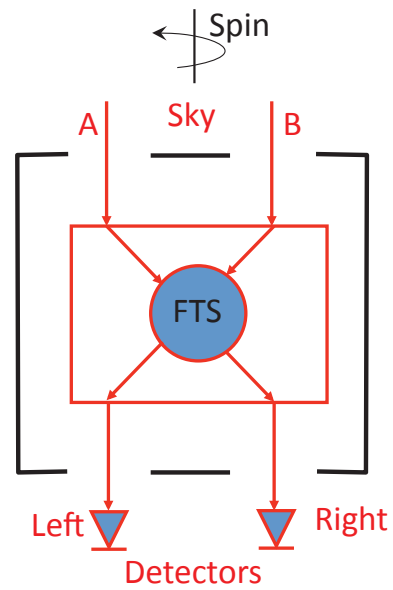

Figure 1. PIXIE's polarizing Fourier Transform Spectrometer operates as a four-port device with two input ports open to the sky and two output ports terminated by polarization-sensitive detectors. Interfering the two beams cancels the effects of common mode beam ellipticity. 
The beam response of a four-port system is considerably more complicated than the two-port systems commonly used for CMB measurements. The double differential nature of the four-port measurement mitigates common spin-dependent polarization errors while providing a means to identify and correct residual effects. Figure 2 shows the PIXIE optical path. Consider (in a time-reversed sense) the path through the optics taken by photons leaving the $\hat{x}$ detector in the left-side concentrator. Since this detector is sensitive to a single linear polarization, the photons exiting the left-side concentrator are entirely in the $\hat{x}$ polarization. A series of polarizing wire grids within the FTS splits the beam and rotates the polarization so that half the initial power exits through port $\mathrm{A}$ in the $\hat{y}$ polarization while the other half exits through port B in the $\hat{x}$ polarization (see, e.g., Appendix A of reference 14). A set of reflective mirrors then couples ports A and B to the sky while preserving the polarization state.

Let us define the beam pattern of the concentrator as $H_{x}(\theta, \phi)$ for the $\hat{x}$ polarization and $H_{y}(\theta, \phi)$ for the $\hat{y}$ polarization, where the angular coordinates $\theta$ and $\phi$ are referred to the sky. Similarly, we define the beam pattern for the fore-optics as $F_{x}(\theta, \phi)$ and $F_{y}(\theta, \phi)$. Using subscripts $L$ and $R$ to distinguish the two concentrator ports and $A$ and $B$ for the two fore-optic ports, we may re-write Equation 2 as

$$
\begin{aligned}
P_{L x} & \propto H_{L x}\left[F_{A x} E_{x}^{2}-F_{B y} E_{y}^{2}\right] \\
P_{L y} & \propto H_{L y}\left[F_{A y} E_{y}^{2}-F_{B x} E_{x}^{2}\right] \\
P_{R x} & \propto H_{R x}\left[F_{B x} E_{x}^{2}-F_{A y} E_{y}^{2}\right] \\
P_{R y} & \propto H_{R y}\left[F_{B y} E_{y}^{2}-F_{A x} E_{x}^{2}\right],
\end{aligned}
$$

where for clarity we suppress the dependence on angular coordinates $(\theta, \phi)$ as well as the phase delay integral over frequency. Two points are apparent. First, the signal at any single detector depends on the convolution of the concentrator beam profile with the differential beam profile generated by the A- and B-side fore-optics. To the extent that the A- and B-side optics have identical beam patterns, the detectors produce no response from an unpolarized sky, regardless of the intensity gradient on the sky or the ellipticity of the fore-optics. This common mode cancellation is performed optically, prior to detection, and does not depend on the instrument calibration. Second, the beam pattern for the concentrator horn appears only as a common-mode multiplicative
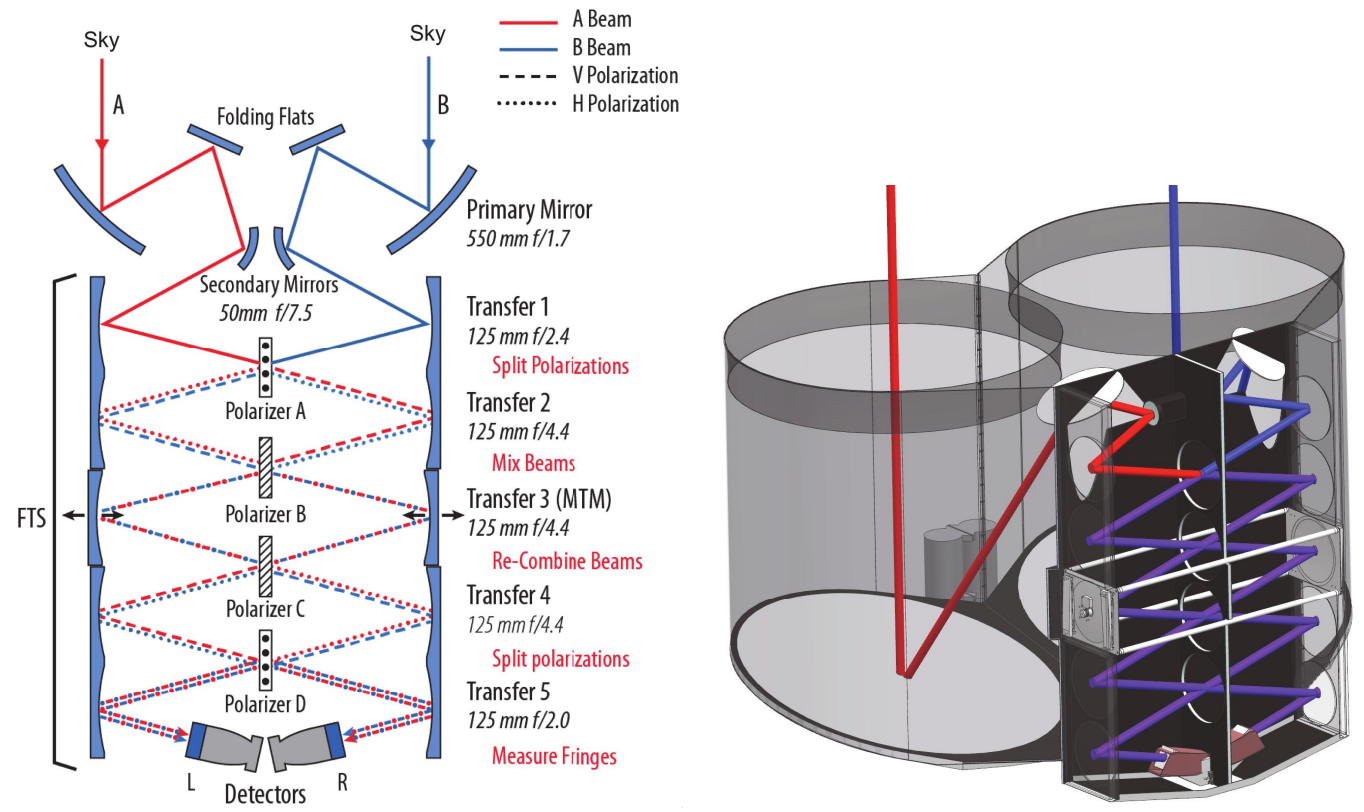

Figure 2. PIXIE optical signal path. The left panel shows the Fourier Transform Spectrometer while the right panel shows the physical layout including the fore-optics. 


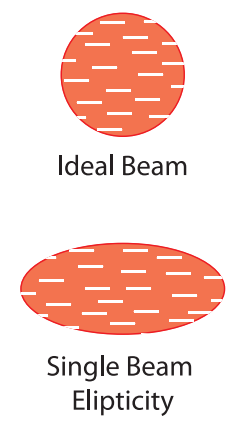

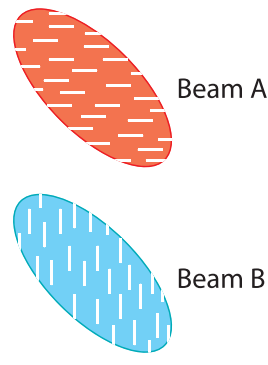

Common Mode

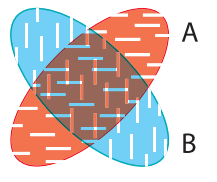

Differential Elipticity
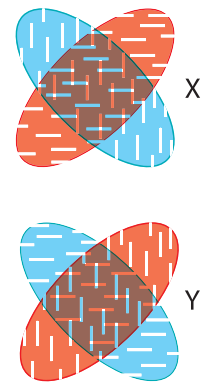

Second Order Cancelling

Figure 3. Cartoon illustrating signal cancellation from differential beam profiles.

factor. Systematic errors coupling temperature anisotropy to polarization depend only on the (highly symmetric) fore-optics and are independent of the concentrator.

Figure 3 illustrates the common-mode subtraction. The left column compares an ideal beam to an elliptical beam in a single-beamed instrument sensitive to a single linear polarization. Rotation of the elliptical beam couples to unpolarized gradients in the sky to produce a time-dependent signal degenerate with a true polarization signal. The second column compares two PIXIE beams, each sensitive to an orthogonal linear polarization (Eq. 2 ). If the beams are identical (common mode only), there is no response to unpolarized sky signal. The third column shows the differential ellipticity between the $\hat{x}$ polarization of the A-side beam and the $\hat{y}$ polarization for the B-side beam. Temperature-polarization mixing for a single detector depends on the differential ellipticity between the A- and B-side beams, and thus appears at second order in the beam differences. Finally, recall that each concentrator contains two detectors sensitive to orthogonal polarization states (Eq. 2). If the differential ellipticity is similar for each detector pair (which view the same sky through the same optics), the beam errors cancel to second order in the co-added signal. Alternatively, we may choose linear combinations of detector output to cancel the common-mode sky signal, thereby isolating beam effects. Such measurements can be used both as confirmation of the expected amplitude of the beam differences and to correct residual beam effects in the sky data.

The double-differential beam cancellation of PIXIE's four-port optical system reduces the sensitivity to unpolarized gradients on the sky. The following sections use Monte Carlo ray-trace code to evaluate the common-mode and differential beam patterns. We quantify the expected systematic error response for ideal optics and show the minimal degradation in performance after accounting for machining and assembly tolerances.

\section{SINGLE-DETECTOR RESPONSE}

Systematic errors in the PIXIE four-port optical system depend on successive differences in the beam patterns. We may write the individual fore-optics beam patterns in terms of the linear combinations

$$
\begin{aligned}
F & =\left(F_{A x}+F_{A y}+F_{B x}+F_{B y}\right) / 4 \\
\Delta & =\left(F_{A x}+F_{A y}-F_{B x}-F_{B y}\right) / 4 \\
\delta & =\left(F_{A x}-F_{A y}+F_{B x}-F_{B y}\right) / 4 \\
\epsilon & =\left(F_{A x}-F_{A y}-F_{B x}+F_{B y}\right) / 4
\end{aligned}
$$

to distinguish the common-mode beam pattern $F=F(\theta, \phi)$ from the differential beam patterns $\Delta$ (A-B spatial asymmetry), $\delta(\hat{x}-\hat{y}$ polarization asymmetry), and $\epsilon$ (spatial/polarization cross term). With these definitions, 

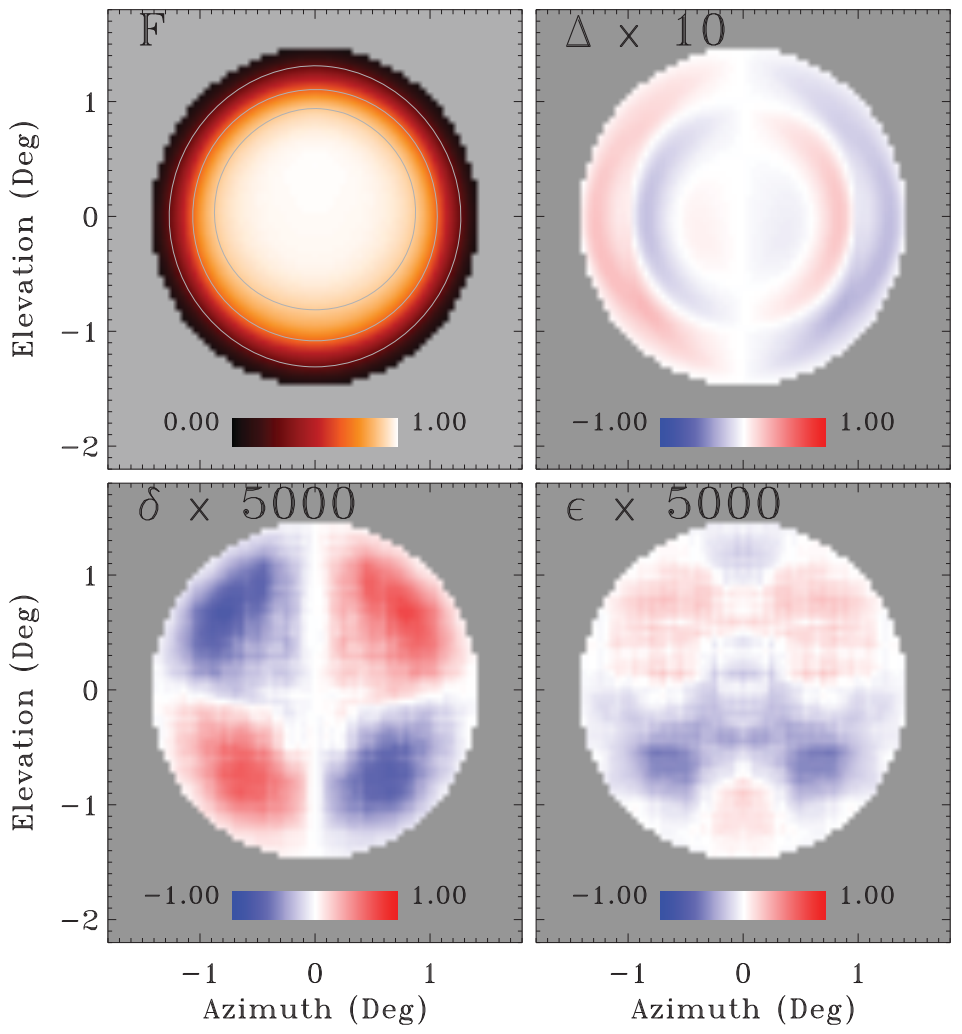

Figure 4. Linear combinations of the PIXIE fore-optics showing the common-mode and differential beam patterns. The spatial $(\Delta)$ and polarization $(\delta)$ asymmetries are small compared to the mean beam pattern $F$. Contours for the commonmode response $F$ are shown at amplitude $0.3,0.7$, and 0.9 to highlight the circular tophat beam structure. Note the change in scale for the three differential beam patterns.

the individual beam patterns become

$$
\begin{aligned}
& F_{A x}=F+\delta+\Delta+\epsilon \\
& F_{A y}=F-\delta+\Delta-\epsilon \\
& F_{B x}=F+\delta-\Delta-\epsilon \\
& F_{B y}=F-\delta-\Delta+\epsilon .
\end{aligned}
$$

Note that these four linear combinations represent a complete set, carrying all information for 2 ports in 2 linear polarizations.

Figure 4 shows the common-mode and differential beam patterns, using a Monte Carlo ray-trace code to propagate $10^{11}$ rays through the PIXIE fore-optics (defined as all elements in the optical chain skyward of the concentrator feed). As expected, the beams are dominated by the common-mode illumination $F$. Out-of-plane reflections at the secondary mirror and folding flat generate a dipolar modulation in the A-B spatial asymmetry $\Delta$, with amplitude 0.03 of the common-mode beam pattern. The polarization asymmetry $\delta$ shows a quadrupolar modulation while the spatial-polarization cross term $\epsilon$ shows higher-order structure, both at amplitude $10^{-4}$ of the common-mode pattern. 
Using these definitions, it is straightforward (if somewhat tedious) to show that

$$
\begin{aligned}
P_{L x} & =H_{L x}[\boldsymbol{Q F}+\boldsymbol{Q} \epsilon+\boldsymbol{I}(\delta+\Delta)] \\
P_{L y} & =H_{L y}[-\boldsymbol{Q} F+\boldsymbol{Q} \epsilon+\boldsymbol{I}(-\delta+\Delta)] \\
P_{R x} & =H_{R x}[\boldsymbol{Q} F-\boldsymbol{Q} \epsilon+\boldsymbol{I}(\delta-\Delta)] \\
P_{R y} & =H_{R y}[-\boldsymbol{Q} F-\boldsymbol{Q} \epsilon+\boldsymbol{I}(-\delta-\Delta)] .
\end{aligned}
$$

The first term in brackets represents the desired polarized sky signal $\boldsymbol{Q}(\theta, \phi)$, convolved with the mean fore-optics beam pattern. The second term, $Q \epsilon$, convolves the true sky polarization with the cross beam pattern $\epsilon(\theta, \phi)$. This term is small. The cross beam pattern may be written as the double difference

$$
\epsilon=\left(F_{A x}-F_{A y}\right)-\left(F_{B x}-F_{B y}\right)
$$

and is thus second order in the beam difference. Furthermore, since this term does not mix the Stokes parameter $\boldsymbol{Q}$ with either $\boldsymbol{U}$ or $\boldsymbol{I}$, it only appears as a scale error in the amplitude of the true sky polarization and may be absorbed by the calibration. The final term represents systematic temperature-polarization coupling.

The left-right symmetry of the PIXIE optics minimizes temperature-polarization coupling. PIXIE's optical design interferes the $\hat{x}$ polarization from one beam with the $\hat{y}$ polarization from the other beam (Eq. 3 ). The optical system is symmetric about the central plane, so that the $\hat{x}$ polarization from one beam is the mirror reflection of the $\hat{y}$ polarization from the other beam (Fig 5). This enforces a reflection symmetry such that

$$
\begin{aligned}
& F_{A x}(\theta, \phi)=F_{B y}(\theta,-\phi) \\
& F_{A y}(\theta, \phi)=F_{B x}(\theta,-\phi)
\end{aligned}
$$

where the azimuthal angle $\phi$ is defined from the midline. Note that this left-right symmetry is not equivalent to an $\hat{x}-\hat{y}$ symmetry since the $\hat{x}-\hat{y}$ coordinate system is rotated by $45^{\circ}$ with respect to the optical midline. Temperature-polarization mixing thus depends on the linear combinations

$$
\begin{aligned}
& \delta+\Delta=F_{A x}-F_{B y} \\
& \delta-\Delta=F_{B x}-F_{A y}
\end{aligned}
$$

proportional to the anti-symmetric component of the difference between the beams.

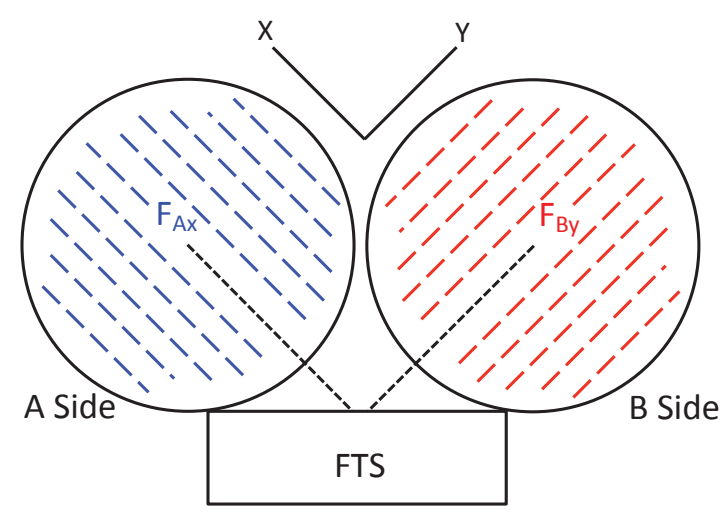

Figure 5. Schematic of the PIXIE optical system showing the symmetric polarization response at the beam apertures. The Fourier transform spectrometer interferes a single linear polarization from one side of the instrument with the orthogonal polarization from the other side. By construction, the $\hat{x}$ polarization on the A side is simply the mirror reflection of the $\hat{y}$ polarization on the B side. 


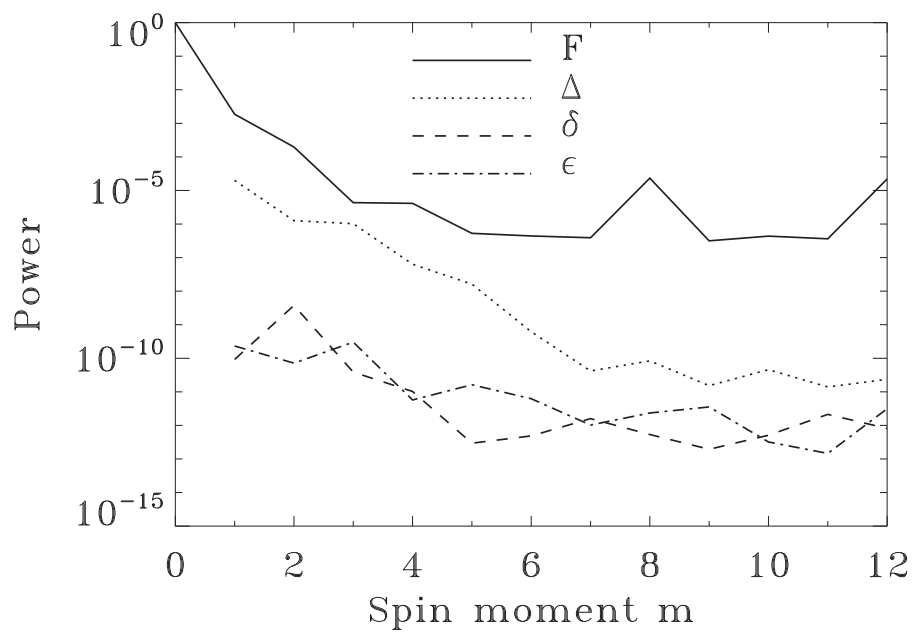

Figure 6. Decomposition of the PIXIE differential beam patterns by spin angle. The common-mode beam $F$ is sensitive only to polarized emission and does not contribute to temperature-polarization systematic errors. The mirror symmetry of the PIXIE optics suppresses temperature-polarization mixing from the A-B spatial asymmetry (beam $\Delta$ ) by a factor of $10^{-6}$ (see text).

The spacecraft spin combines with the mirror symmetry of the instrument optics to further minimize temperature-polarization coupling. Each detector is sensitive to a single linear polarization (Stokes $Q$ in a coordinate system fixed with respect to the instrument). The entire spacecraft rotates about the instrument boresight to interchange the roles of $\hat{x}$ and $\hat{y}$ polarization at the detectors, allowing full characterization of the Stokes $Q$ and $U$ parameters on the sky. True sky polarization is modulated at twice the spacecraft spin frequency,

$$
Q_{\text {inst }}=Q_{\text {sky }} \cos (2 \gamma)+U_{\text {sky }} \sin (2 \gamma) \text {. }
$$

Temperature-polarization mixing is dominated by the anti-symmetric component of the differential beam pattern from the instrument fore-optics. Anti-symmetric signals can only appear at odd harmonics of the spacecraft spin, and may readily be distinguished from true sky polarization.

We quantify the suppression of temperature-polarization systematic errors using the spin-dependent moments of the differential beam patterns. The instantaneous power at each detector depends on the convolution of the beam pattern (in instrument-fixed coordinates) with the sky signal (rotated from sky to instrument coordinates). Azimuthal asymmetry in the beam patterns causes the measured power to vary with the spacecraft spin angle. We thus compute the coefficients

$$
\begin{aligned}
& a_{m}=\int B(\Omega) \cos (m \phi) d \Omega \\
& b_{m}=\int B(\Omega) \sin (m \phi) d \Omega
\end{aligned}
$$

where $B$ represents one of the linear combinations of beam patterns (Eq. 4) and $d \Omega=\sin (\theta) d \theta d \phi$ is computed in instrument coordinates centered on the boresight.

Figure 6 shows the power $P_{m}=a_{m}^{2}+b_{m}^{2}$ as a function of spin moment $m$. The odd-even asymmetry in spin moment $m$ is superposed atop an overall decrease in power with increasing $m$. The noise floor reflects shot noise from the discrete ray-trace simulation. Recall that the common-mode beam pattern $F$ is sensitive only to polarized emission on the sky (Eq. 6) and does not create temperature-polarization errors even for the $m=2$ case. Systematic errors from temperature-polarization coupling are dominated by the A-B spatial asymmetry $\Delta$, and are suppressed by a factor $10^{-6}$ relative to the polarization response in the common-mode beam. 


\section{ADDITIONAL SYMMETRIES}

The mirror symmetry of PIXIE's differential 4-port interferometer suppresses systematic errors from temperaturepolarization coupling by 6 orders of magnitude for the single-detector response. Additional symmetries between different detectors allow further suppression of beam-related systematic errors. The left and right concentrators are identical, resulting in left-right symmetry

$$
\begin{aligned}
& H_{L x}(\theta, \phi)=H_{R x}(\theta,-\phi) \\
& H_{L y}(\theta, \phi)=H_{R y}(\theta,-\phi)
\end{aligned}
$$

for identical polarization states. This is similar to the left-right symmetry in Eq. 8 except that the symmetry is now between identical polarization states on opposite sides of the instrument.

Differences between the two polarizations $\hat{x}$ and $\hat{y}$ within a single concentrator can occur, corresponding to the difference between the E-plane and H-plane beam patterns for a single-moded feed. PIXIE's multi-moded operation reduces this effect, which vanishes in the geometric optics limit. We further reduce the effect by rotating the concentrator so that the symmetry axes of the square aperture lie at $\pm 45^{\circ}$ relative to the $\hat{x}$ and $\hat{y}$ polarization vectors (Fig 7). The resulting beams in $\hat{x}$ and $\hat{y}$ are equivalent linear combinations of the E-plane and H-plane beam patterns, so that

$$
\begin{aligned}
& H_{L x} \approx H_{L y} \\
& H_{R x} \approx H_{R y}
\end{aligned}
$$

with residuals resulting from small displacements in the rotation angle. ${ }^{15}$ Without loss of generality, we may follow Eq. 5 to decompose the beam pattern from each horn into a component common to all four detectors plus a set of differential beam patterns,

$$
\begin{aligned}
H & =\left(H_{L x}+H_{L y}+H_{R x}+H_{R y}\right) / 4 \\
\rho & =\left(H_{L x}-H_{L y}+H_{R x}-H_{R y}\right) / 4 \\
\tau & =\left(H_{L x}+H_{L y}-H_{R x}-H_{R y}\right) / 4 \\
\kappa & =\left(H_{L x}-H_{L y}-H_{R x}+H_{R y}\right) / 4
\end{aligned}
$$

so that

$$
\begin{aligned}
& H_{L x}=H+\rho+\kappa+\tau \\
& H_{L y}=H-\rho+\kappa-\tau \\
& H_{R x}=H+\rho-\kappa-\tau \\
& H_{R y}=H-\rho-\kappa+\tau
\end{aligned}
$$

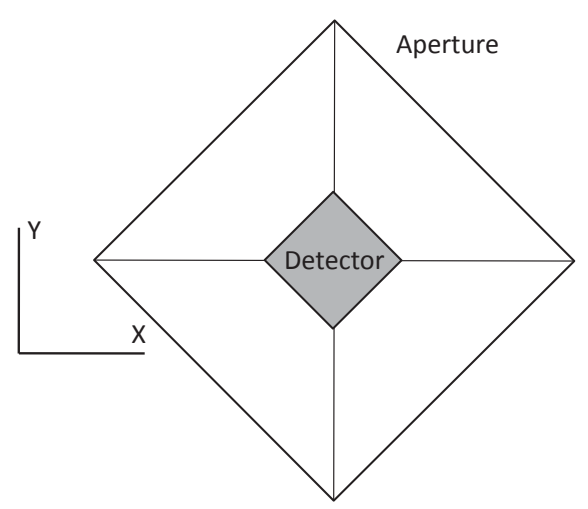

Figure 7. Schematic showing the orientation of the PIXIE concentrator. The square aperture is rotated $45^{\circ}$ to minimize any differences between the $\hat{x}$ and $\hat{y}$ polarization. 

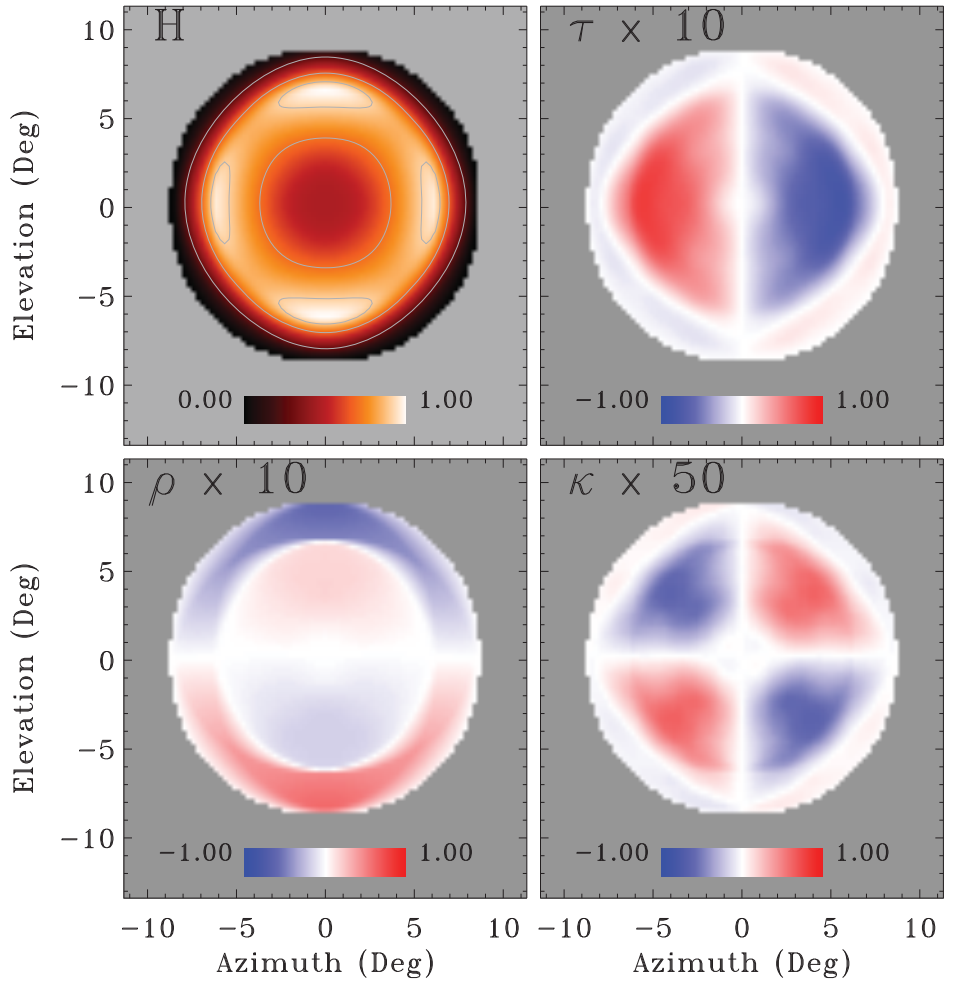

Figure 8. Common-mode and differential beam patterns for the PIXIE feed horn concentrators. The feed horn beam pattern does not directly source $T \rightarrow B$ systematic errors, but only modulates the effect from the differential fore-optics. The off-axis design creates dipolar modulation in the differential beam patterns $\rho$ and $\tau$, while the square shape is reflected in the quadrupolar modulation for $\kappa$. Contours for the common-mode response $H$ are shown at amplitude $0.3,0.7$, and 0.9 .

where the horn parameters are defined analogously to the fore-optics in Eq. 4. Figure 8 shows the common-mode and differential beam patterns from the concentrator horn. Asymmetries from the off-axis orientation appear at the few-percent level. As with the fore-optics, the differential beams are dominated by an anti-symmetric (dipolar) component.

\section{COMBINED DETECTOR RESPONSE}

PIXIE's four detectors share different portions of the optical system (left or right concentrator, $\hat{x}$ or $\hat{y}$ polarization). Linear combinations of the post-detection signals can either eliminate or isolate specific systematic error signals, providing additional safeguards against temperature-polarization mixing. For example, we may combine all 4 detectors to yield the sum signal

$$
\begin{aligned}
{\left[P_{L x}-P_{L y}+P_{R x}-P_{R y}\right] / 4 } & =\boldsymbol{Q} H F \\
& +\boldsymbol{Q} \epsilon \tau \\
& +\boldsymbol{I} H \delta \\
& +\boldsymbol{I} \Delta \tau
\end{aligned}
$$

As before, the first term is the true sky polarization, now convolved with the combined common-mode beam pattern from the feed horn and fore-optics. The second term affects only the amplitude of the true sky polarization and may be absorbed into the calibration. The final two terms represent systematic errors coupling temperature anisitropy to polarization. 
Table 1. Common-Mode and Differential Beam Patterns

\begin{tabular}{lcccc}
\hline \hline & \multicolumn{4}{c}{ Fore-Optics } \\
Parameter & $F$ & $\Delta$ & $\delta$ & $\epsilon$ \\
\hline Peak Amplitude & $1.0 \mathrm{E}+00$ & $2.9 \mathrm{E}-02$ & $1.5 \mathrm{E}-04$ & $1.0 \mathrm{E}-04$ \\
Solid Angle (sr) & $1.3 \mathrm{E}-03$ & $2.0 \mathrm{E}-05$ & $1.1 \mathrm{E}-07$ & $5.1 \mathrm{E}-08$ \\
Relative Beam Area $f$ & $1.0 \mathrm{E}+00$ & $1.5 \mathrm{E}-02$ & $8.1 \mathrm{E}-05$ & $3.8 \mathrm{E}-05$ \\
Power $(\mathrm{m}=1)$ & $7.0 \mathrm{E}-05$ & $1.8 \mathrm{E}-05$ & $9.3 \mathrm{E}-11$ & $2.3 \mathrm{E}-10$ \\
Power $(\mathrm{m}=2)$ & $3.0 \mathrm{E}-04$ & $1.4 \mathrm{E}-06$ & $3.8 \mathrm{E}-09$ & $7.2 \mathrm{E}-11$ \\
\hline \hline & & Horn Concentrator & \\
Parameter & $H$ & $\rho$ & $\tau$ & $\kappa$ \\
\hline Peak Amplitude & $1.0 \mathrm{E}+00$ & $6.1 \mathrm{E}-02$ & $8.2 \mathrm{E}-02$ & $1.3 \mathrm{E}-02$ \\
Solid Angle (sr) & $4.3 \mathrm{E}-02$ & $1.3 \mathrm{E}-03$ & $1.9 \mathrm{E}-03$ & $3.2 \mathrm{E}-04$ \\
Relative Beam Area $f$ & $1.0 \mathrm{E}+00$ & $3.0 \mathrm{E}-02$ & $4.5 \mathrm{E}-02$ & $7.4 \mathrm{E}-03$ \\
Power $(\mathrm{m}=1)$ & $4.8 \mathrm{E}-08$ & $1.5 \mathrm{E}-04$ & $9.5 \mathrm{E}-04$ & $3.9 \mathrm{E}-08$ \\
Power $(\mathrm{m}=2)$ & $6.4 \mathrm{E}-04$ & $8.4 \mathrm{E}-10$ & $3.7 \mathrm{E}-06$ & $2.7 \mathrm{E}-05$ \\
\hline \hline
\end{tabular}

We use Monte Carlo ray-trace simulations to quantify the expected amplitude of these terms. Table 1 summarizes the common-mode and differential beam patterns for the PIXIE optical system. The differential beam patterns are small compared to the common-mode response. We compare the weighted beam area of the differential beams to the weighted area of the common-mode beam pattern,

$$
f=\frac{\int|\Delta(\theta, \phi)| d \Omega}{\int|F(\theta, \phi)| d \Omega},
$$

computed similarly for each of the 6 differential beam patterns. The differential beams have fractional area of a few percent for the concentrator, and $10^{-2}$ to $10^{-5}$ for the more symmetric fore-optics. The differential beams are dominated by a dipolar modulation $(m=1)$ which does not lead to temperature-polarization mixing. The systematic error response to spin modulation at $m=2$ is typically of order $10^{-6}$ or smaller.

We may now quantify the systeatic error terms in the post-detection linear combination. The third term $\boldsymbol{I} H \delta$ is similar to the temperature-polarization mixing $\boldsymbol{I} H \Delta$ from a single detector (eq. 9), but reduced in amplitude by a factor of 200 due to replacing the A-B differential beam pattern $\Delta$ with the smaller $\hat{x}-\hat{y}$ differential beam pattern $\delta$. The lower response to $m=2$ modulation from the $\delta$ differential beam (compared to the $\Delta$ beam) produces additional systematic error suppression. The final term $\boldsymbol{I} \Delta \tau$ also represents temperature-polarization mixing, but now appears at second order in small beam differences and is reduced by a factor 20 in amplitude from the single-detector error. The $m=2$ spin modulation of the $\tau$ differential beam yields additional suppression.

We may also choose linear combinations of detectors to cancel the sky signal $\boldsymbol{Q} H F$, thereby isolating different systematic error signals. Such measurements of the systematic error signals can be used both to correct the sky measurements and as confirmation of the expected effect from beam pattern differences. For example, the orthogonal combination of four detectors becomes

$$
\begin{aligned}
{\left[P_{L x}-P_{L y}-P_{R x}+P_{R y}\right] / 4 } & =\boldsymbol{Q} F \kappa \\
& +\boldsymbol{Q} \epsilon \tau \\
& +\boldsymbol{I} \Delta \rho \\
& +\boldsymbol{I} \delta \kappa
\end{aligned}
$$

We may again use Table 1 to estimate the amplitude of each term. Unpolarized CMB signals $\boldsymbol{I}$ have amplitude of order $100 \mu \mathrm{K}$, while E-mode polarization $\boldsymbol{Q}$ is of order $1 \mu \mathrm{K}$. Multiplying each CMB term by the relative beam area of each beam pattern yields an estimate of the relative amplitude of each term (prior to spin modulation). The difference signal is dominated by the term $\boldsymbol{I} \Delta \rho$, representing the convolution of the unpolarized CMB anisotropy with the double beam difference $\Delta \rho$. We may instead choose to compare signals from the two detectors 

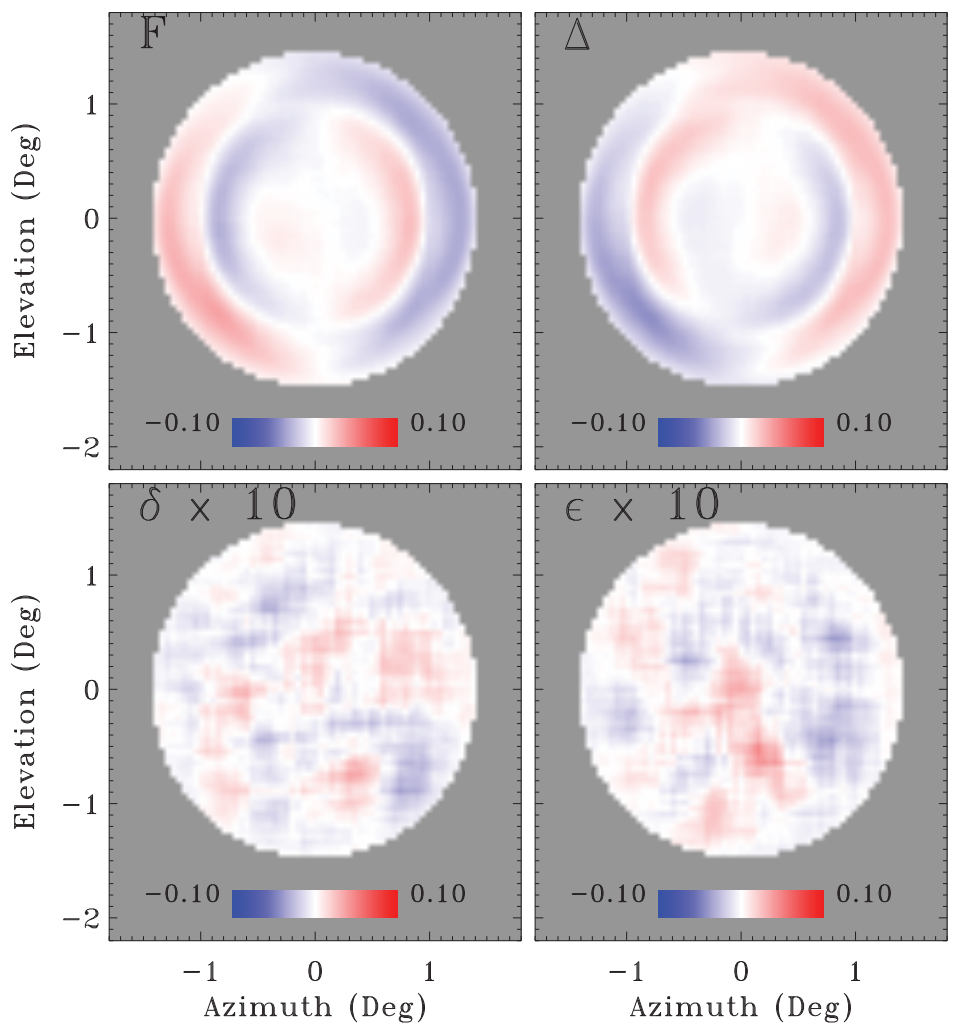

Figure 9. Differences between the nominal beam patterns from Figure 4 and the distorted beam patterns after allowing for machining and assembly tolerances. Beam patterns are shown from a single Monte Carlo realization in which the position and orientation of each optical element are perturbed about the nominal configuration.

sharing a common concentrator. A similar analysis shows that the detector-pair combination $\left(P_{L x}+P_{L y}\right) / 2$ is dominated by the term $\boldsymbol{I} H \Delta$ which isolates a single differential beam for measurement and correction. Similar linear combinations can isolate other terms.

\section{TOLERANCE}

Mirror symmetries within PIXIE's differential optics suppress systematic errors coupling unpolarized structure in the sky to a false polarized signal. Figure errors in the optical components during the machining process, as well as positioning errors during assembly, distort the beams from the ideal beam patterns. We quantity the degradation in optical performance using 30 Monte Carlo realizations of the PIXIE optical system. For each realization, we adjust the position of each optical element allowing both translation and rigid-body rotation assuming assembly and machining tolerances of $\pm 0.05 \mathrm{~mm}$ drawn from a random Gaussian distribution. After adjusting all optical elements, we follow the paths of $10^{9}$ rays through the adjusted optical system to define the distorted beam patterns.

The PIXIE optical system is robust to typical machining and assembly tolerances. Figure 9 shows the difference between the nominal beam patterns and the distorted patterns for a single Monte Carlo realization of the distorted optical system. The dominant effect is an angular displacement of the beams of order $3^{\prime}$, creating an anti-symmetric pattern in both the mean beam $(F)$ and the A-B spatial asymmetry $(\Delta)$. Angular displacement of the beam centroid couples to spin moment $m=1$ and does not induce additional temperature-polarization mixing. 


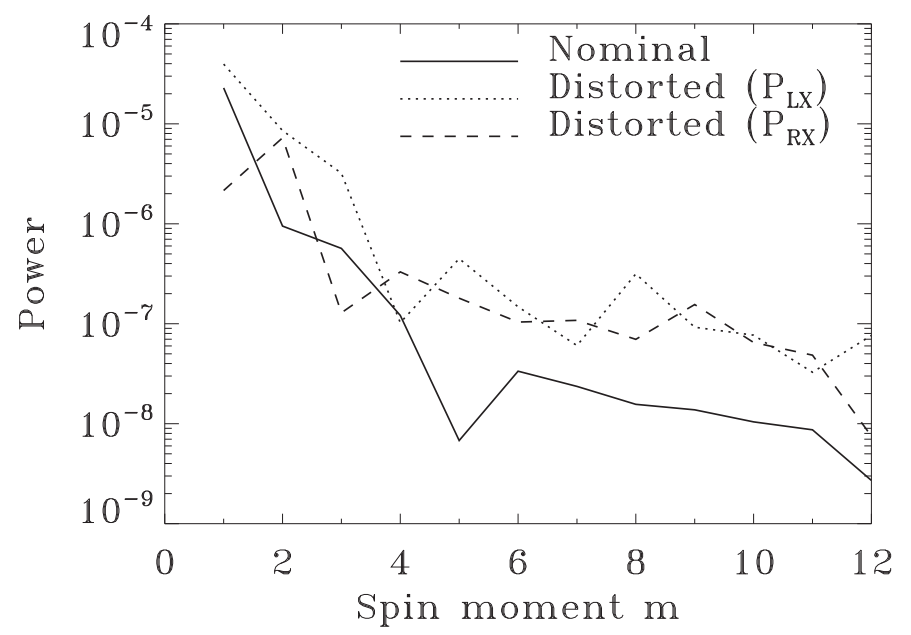

Figure 10. Effects of machining and assembly tolerances on the differential beam pattern $\Delta(\theta, \phi)$. We compare the differential beam pattern for the nominal optical configuration to a Monte Carlo realization with all optical elements perturbed from their nominal positions. The distorted patterns are shown as a function of spin moment $m$ for the detectors sensitive to $\hat{x}$ sky polarization in both the left-side and right-side concentrators. The distorted optical system still shows suppression of order $10^{-5}$ for temperature-polarization coupling at $m=2$.

Figure 10 compares the spin dependence of the nominal beam patterns to the distorted beams generated from a single Monte Carlo realization of the full optical system. It is similar to the ideal beam patterns shown in Figure 6, except that the position and pointing of each optical element has been perturbed by an amount randomly chosen from a Gaussian normal distribution of width $0.05 \mathrm{~mm}$. We now also include the illumination of the (perturbed) fore-optics by the (perturbed) concentrator. For clarity, we compare the spin decomposition for the nominal and distorted configurations for a single choice of differential beam. Temperature-polarization coupling is dominated by the $\mathrm{A}-\mathrm{B}$ differential beam $\Delta(\theta, \phi)$. PIXIE has 4 detectors; we show the distorted beam decomposition for detectors observing the same $(\hat{x})$ sky polarization from either the left or right concentrator. The distorted optical system has a larger response to systematic error coupling at $m=2$, but the response is still suppressed by a factor of $10^{5}$ compared to the true sky polarization.

\section{DISCUSSION}

Systematic errors coupling unpolarized anisotropy to a false polarized signal are a common concern to CMB polarimeters. PIXIE's optical design provides several layers of mitigation compared to instruments imaging the CMB across a large (kilo-pixel) focal plane. Missions employing kilo-pixel arrays across large fields of view must account for the systematic degradation in beam shape from coma and shear for detectors farther from the center of the focal plane. All four PIXIE detectors, in contrast, lie at the center of the focal plane, allowing ellipticity to be minimized (Fig 4).

More importantly, PIXIE's four-port optical system provides three distinct levels of differential measurement. The Fourier transform spectrometer produces a signal that depends on the difference between two nearly-identical beams on the sky. This differential measurement is performed optically, prior to detection, and is independent of detector calibration. We use ray-trace simulations to evaluate the differential beam patterns after removing the common-mode response. The differential beams can be described in terms of the spatial asymmetry between the A- and B-sides of the instrument, the polarization asymmetry between the $\hat{x}$ and $\hat{y}$ response, plus a cross term for the mixed spatial-polarization difference. All of the differential beams are small compared to the common-mode response. The largest effect is the spatial (A-B) asymmetry, which has only $1.5 \%$ of the common-mode response. The other differential beams have response below $0.01 \%$.

PIXIE's symmetric design further reduces systematic error response from the differential beam cancellation. The FTS interferes the $\hat{x}$ polarization from the A-side beam with the $\hat{y}$ polarization from the B-side beam (Fig 
5). The optical system is symmetric about the mid-plane between the two sides, which forces the $\hat{x}$ polarization from one beam to be the mirror reflection of the $\hat{x}$ polarization from the other beam. The $\mathrm{A}-\mathrm{B}$ mirror reflection combines with the A-B beam subtraction to produce an anti-symmetric (dipole) response in the differential beam patterns. The anti-symmetric part of the differential beam pattern does not contribute to the systematic error from temperature-polarization coupling. Each detector samples a single polarization state; the entire instrument spins about the boresight to allow full sampling of the sky polarization. True polarized signals appear at twice the spin frequency, while anti-symmetric signals can only appear at odd harmonics of the spin. Systematic errors from temperature-polarization coupling thus depend only on the $m=2$ component of the differential beam patterns, which are dominated by a dipole $(m=1)$ response. Ray-trace models of the PIXIE beams show that the response at $m=2$ is reduced by an additional factor of $10^{6}$ or more. In principle, the optical system could further be optimized to suppress the $m=2$ differential beam response, moving power to other $m$ values that do not participate in temperature-polarization mixing. This has not yet been done.

Finally, we may follow the common practice for CMB measurements and combine the post-detection signals from individual detectors. The four detectors are mounted in identical concentrators and view the same sky direction through the same optical path. Combining all four detectors cancels the leading effects from differential beams in the single-detector signal, reducing the systematic error response by a factor of 1000 or more compared to the individual detectors. Conversely, orthogonal linear combinations of 2 or 4 detectors can cancel the polarized sky signal to isolate, identify, and model specific systematic effects from the individual differential beam patterns.

Systematic error suppression in the differential PIXIE optics is robust against typical machining and assembly tolerances. We combine ray-trace optical simulations with Monte Carlo realizations of distorted PIXIE optics to evaluate both the individual beam patterns and the resulting systematic error response. Each Monte Carlo realization of then PIXIE optics perturbs each optical element (mirrors, folding flats, polarizing grids, etc) in both position and orientation by an amount drawn from a Gaussian distribution whose width is set by typical machining/assembly tolerances of $0.05 \mathrm{~mm}$. The dominant effect of such tolerance errors is an angular displacement of the A-side beams relative to the B-side beams. The two beams are normally co-pointed on the sky; after accounting for tolerances them beams are typically mis-aligned by $3^{\prime}$. This is small compared to the $2.6^{\circ}$ width of the common-mode beams; the resulting dipolar beam asymmetries predominantly effect the $m=1$ spin moment and do not couple efficiently to polarization. The distorted optical system still provides suppression of the $m=2$ temperature- polarization systematic error by factor of order $10^{6}$.

\section{REFERENCES}

[1] Rubakov, V. A., Sazhin, M. V., and Veryaskin, A. V., "Graviton creation in the inflationary universe and the grand unification scale," Physics Letters B 115, 189-192 (Sept. 1982).

[2] Fabbri, R. and Pollock, M. D., "The effect of primordially produced gravitons upon the anisotropy of the cosmological microwave background radiation," Physics Letters B 125, 445-448 (June 1983).

[3] Abbott, L. F. and Wise, M. B., "Constraints on generalized inflationary cosmologies," Nuclear Physics B 244, 541-548 (Oct. 1984).

[4] Polnarev, A. G., "Polarization and anisotropy induced in the microwave background by cosmological gravitational waves," Astronomicheskii Zhurnal 62, 1041-1052 (Dec. 1985).

[5] Davis, R. L., Hodges, H. M., Smoot, G. F., Steinhardt, P. J., and Turner, M. S., "Cosmic microwave background probes models of inflation," Physical Review Letters 69, 1856-1859 (Sept. 1992).

[6] Grishchuk, L. P., "Cosmological perturbations of quantum-mechanical origin and anisotropy of the microwave background," Physical Review Letters 70, 2371-2374 (Apr. 1993).

[7] Kamionkowski, M., Kosowsky, A., and Stebbins, A., "Statistics of cosmic microwave background polarization," Physical Review D 55, 7368-7388 (June 1997).

[8] Seljak, U. and Zaldarriaga, M., "Signature of Gravity Waves in the Polarization of the Microwave Background," Physical Review Letters 78, 2054-2057 (Mar. 1997).

[9] Lyth, D. H. D. H. and Riotto, A. A., "Particle physics models of inflation and the cosmological density perturbation," Physics Reports 314, 1-146 (June 1999). 
[10] Hu, W., Hedman, M. M., and Zaldarriaga, M., "Benchmark parameters for CMB polarization experiments," Physical Review D, eprint = astro-ph/0210096, keywords = Background radiations, year = 2003, month = feb, volume $=67$, number $=4$, eid $=043004$, pages $=043004$, doi $=10.1103 /$ PhysRevD.67.043004, adsurl $=$ http://adsabs.harvard.edu/abs/2003PhRvD..67d3004H, adsnote = Provided by the SAO/NASA Astrophysics Data System .

[11] O'Dea, D., Challinor, A., and Johnson, B. R., "Systematic errors in cosmic microwave background polarization measurements," Monthly Notices of the Royal Astronomical Society 376, 1767-1783 (Apr. 2007).

[12] Rosset, C., Yurchenko, V. B., Delabrouille, J., Kaplan, J., Giraud-Héraud, Y., Lamarre, J.-M., and Murphy, J. A., "Beam mismatch effects in cosmic microwave background polarization measurements," Astronomy and Astrophysics 464, 405-415 (Mar. 2007).

[13] Shimon, M., Keating, B., Ponthieu, N., and Hivon, E., "CMB polarization systematics due to beam asymmetry: Impact on inflationary science," Physical Review D 77, 083003 (Apr. 2008).

[14] Kogut, A., Fixsen, D. J., Chuss, D. T., Dotson, J., Dwek, E., Halpern, M., Hinshaw, G. F., Meyer, S. M., Moseley, S. H., Seiffert, M. D., Spergel, D. N., and Wollack, E. J., "The Primordial Inflation Explorer (PIXIE): a nulling polarimeter for cosmic microwave background observations," Journal of Cosmology and Astroparticle Physics 7, 25 (July 2011).

[15] Kogut, A. J. and Fixsen, D. J., "Polarized beam patterns from a multimoded feed for observations of the cosmic microwave background," Journal of Astronomical Telescopes, Instruments, and Systems 4, 014006 (Jan. 2018). 